\title{
Extracellular and intracellular microRNAs in pancreatic cancer: from early diagnosis to reducing chemoresistance
}

\author{
Xiaohong Jiang ${ }^{*}$, Dongxia Hou ${ }^{\dagger}$, Zhe Wei, Shasha Zheng, Yujing Zhang ${ }^{*}$ and Jing Li ${ }^{*}$
}

\begin{abstract}
Pancreatic cancer $(\mathrm{PaC})$ is one of the most aggressive malignancies. The dismal survival rate of $\mathrm{PaC}$ patients is mainly due to the late diagnosis and their resistance to conventional chemotherapies. Emerging evidence suggests that miRNA can serve as a potential new diagnostic and therapeutic weapon to fight against PaC. Circulating miRNAs represent the most promising noninvasive tools for diagnosis owing to their high stability in blood. Combinations of circulating miRNAs with other serum indicators such as carbohydrate antigen 19-9 (CA19-9) were demonstrated to be valuable biomarkers for early PaC diagnosis. As miRNAs can regulate epithelial-mesenchymal transition (EMT) and the progression of cancer stem cells (CSCs), two critical factors in PaC drug resistance, selectively manipulating miRNAs may improve the sensitivity of certain $\mathrm{PaC}$ chemotherapeutic agents, such as gemcitabine. Therefore, the investigations of miRNAs in $\mathrm{PaC}$ may provide potential novel approaches for both tumor diagnosis and treatment.
\end{abstract}

Keywords: Pancreatic cancer, Circulating microRNAs, Biomarkers, Epithelial-mesenchymal transition, Chemoresistance

\section{Introduction}

Pancreatic Cancer $(\mathrm{PaC})$ is currently the 3rd cause of cancer-associated deaths in the U.S. surpassing breast cancer [1], with $\sim 53,670$ new diagnoses (greater than $90 \%$ is pancreatic ductal adenocarcinoma, PDAC) and 43,090 deaths predicted to occur in 2017 [2, 3]. Only $24 \%$ of patients survive for 1 year after diagnosis with $\mathrm{PaC}$, even in countries with the best standard of care [4]. Currently, owning to no effective early screening test, about $80 \%$ of $\mathrm{PaC}$ patients are too late to do the potentially curative resection [5]. In addition, $\mathrm{PaC}$ is highly resistant to conventional chemotherapies (gemcitabine), which led to the high mortality of $\mathrm{PaC}$ patients. According to clinical trials, conventional chemotherapy treatment only increase 5 weeks of survival in patients diagnosed with advanced $\mathrm{PaC}[6,7]$. Therefore, the patients presenting with advanced $\mathrm{PaC}$ have extremely low

\footnotetext{
*Correspondence: xiaohongjiang@nju.edu.cn; yjzhang@nju.edu.cn; jingli220@nju.edu.cn

${ }^{+}$Xiaohong Jiang and Dongxia Hou contributed equally to this work. State Key Laboratory of Pharmaceutical Biotechnology, Collaborative Innovation Center of Chemistry for Life Sciences, Jiangsu Engineering Research Center for MicroRNA Biology and Biotechnology, NJU Advanced Institute for Life Sciences (NAILS), School of Life Sciences, Nanjing University, 163 Xianlin Avenue, Nanjing 210046, Jiangsu, China
}

survival times, underlines the urgent need to improve both early diagnosis and further understanding of drug-resistant mechanisms of $\mathrm{PaC}$.

MicroRNA (miRNA) is a class of small noncoding RNAs that negatively regulate target gene expression at post-transcriptional level $[8,9]$. As tiny but powerful players in cell regulation, miRNAs are almost involved in all biologic processes in mammals [10,11]. Aberrant miRNA expressions are observed in $\mathrm{PaC}$, thus, targeting miRNAs may provide fundamentally new approaches to reduce chemoresistance in $\mathrm{PaC}$. Moreover, studying of the stably expressed circulating miRNAs in blood may provide us a gold mine of noninvasive biomarkers in cancer [10]. Since the change of miRNA expressions usually occurs during early tumorigenesis, we thus describe the possibility of using circulating miRNAs for early $\mathrm{PaC}$ detection, which can be applicable diagnostic and prognostic markers in $\mathrm{PaC}$ [12-15]. The roles of miRNAs in epithelial-mesenchymal transition (EMT), cancer stem cells (CSCs), and their biological significances in $\mathrm{PaC}$ and possible applications to reduce chemoresistance are also discussed.

(c) The Author(s). 2019 Open Access This article is distributed under the terms of the Creative Commons Attribution 4.0 International License (http://creativecommons.org/licenses/by/4.0/), which permits unrestricted use, distribution, and reproduction in any medium, provided you give appropriate credit to the original author(s) and the source, provide a link to the Creative Commons license, and indicate if changes were made. The Creative Commons Public Domain Dedication waiver (http://creativecommons.org/publicdomain/zero/1.0/) applies to the data made available in this article, unless otherwise stated. 


\section{Extracellular miRNAs: Potential biomarkers for early detection of $\mathrm{PaC}$ \\ Current diagnosis of $\mathrm{PaC}$}

'The silent killer' $\mathrm{PaC}$ often shows no symptoms in the early stages unless the primary tumor is located in the pancreas head [16, 17]. This leads to PaC being diagnosed until when it has spread beyond the pancreas itself [5]. Clinical trials showed that for the patients who are able to remove their pancreatic tumor, their 5-year survival rate are significantly improved by adjuvant chemotherapy [18-20]. Unfortunately, when diagnosis, only less than $15 \%$ of patients were surgically resectable [21].

Abdominal pain, unusual bloating, belching, heartburn, altered bowel habits, symptoms of biliary obstruction are the most commonly symptoms before $\mathrm{PaC}$ diagnosis, unfortunately, usually only advanced $\mathrm{PaC}$ exerts enough specific symptoms [22]. Therefore, early detection of $\mathrm{PaC}$ is urgently needed [23]. Current noninvasive imaging techniques such as ultrasound, contrast-enhanced multidetector CT, and MRI are unable to detect tumor $<1-2 \mathrm{~cm}$ in size [24]. Carbohydrate antigen 19-9 (CA19-9), the most extensively used biomarker in $\mathrm{PaC}$ diagnosis, is also used to predict tumor recurrence $[25,26]$. However, it still lack of sensitivity, and often shows false-positive elevation in the presence of obstructive jaundice [25]. Thus, it is urgent to devise better diagnostic markers for PaC. Despite a large number of potential markers have been identified in $\mathrm{PaC}$, such as cytokeratin, glycoprotein, few have proven advantageous when compared to the currently used CA19-9 serum testing [25, 27-32].

Over the past decade, miRNAs were found to be important regulators in carcinogenesis process [11]. Many studies have demonstrated that miRNAs were either oncogenic or acted as tumor-suppressors [33]. Due to their stability in fresh and formalin-fixed paraffin-embedded samples, the deregulated tissue miRNAs represent feasible diagnostic or prognostic markers for $\mathrm{PaC}[34,35]$.

Currently, the noninvasive blood-based test is still the most convenient early diagnostic approach. In addition, the ideal blood $\mathrm{PaC}$ biomarkers would allow for diagnosis before it spreads to other organs $[16,36]$. In this part, we focus on the amazing discovery that a large amount of miRNAs are stably expressed in the circulation, which might provide an easy and promising early diagnosis strategy for $\mathrm{PaC}$ [11, 37-39]. Studies by several independent groups clearly show that the circulating miRNAs are protected from endogenous ribonuclease activity because binding to proteins [40-42], or being packed by secretory exosomes $[43,44]$.

\section{Single circulating miRNA as potential biomarker}

Allen et al. first found that circulating miR-210 was significantly elevated in plasma samples from $\mathrm{PaC}$ patients [45]. In the same year Ang et al. reported that both tissue and serum miR-200a/b were up-regulated in $\mathrm{PaC}$ patients [46]. As shown in Table 1, identification of these circulating miRNA-based biomarkers opens up a promising field of using the expression profile of circulating miRNAs for PaC diagnosis. Otsuji's group found that the miR-18a and miR-221, which belong to the oncogenic miR-17/92 and miR-221/222 clusters, were highly expressed in both $\mathrm{PaC}$ tissue and plasma samples [47-49]. Zhang et al. [50, 51] demonstrated that the circulating miR-192 and miR-194 in serum may be potential sensitive diagnostic biomarkers for PDAC. Kong et al. showed that serum miR-196a could be used to select possible surgical candidates, because elevated miR-196a level was closely related to poor survival $\mathrm{PaC}[52,53]$. Their results were proven later by Bartsch and co-workers [54]. Sun et al. also revealed that downregulation of serum of miR-124 was linked to the poor prognosis in patients with PDAC [55]. Michael et al. found that the elevated level of serum miR-1290 could sensitively distinguish patients with low-stage PaC from controls [56]. Tessa et al. showed that circulating miR-485-3p and miR-938 could discriminate PDAC patients from healthy individuals and patients with chronic pancreatitis (CP) [57-59]. miR-25 has also been demonstrated to be a potential novel biomarker for the early $\mathrm{PaC}$ diagnosis [60]. By analyzing the expression levels of 6 miRNAs that up-regulated in PDAC, Alemar et al. showed that miR-21 and miR-34a are potentially useful in diagnosing PDAC [61]. By assessing miR-182 in 109 $\mathrm{PaC}$ and $38 \mathrm{CP}$ as well as 50 healthy controls, Chen et al. suggested that miR-182 may be a potential marker for both diagnosis and prognosis of $\mathrm{PaC}$, with a sensitivity of $64.1 \%$ and a specificity of $82.6 \%$ [62].

\section{Panels of circulating miRNAs as potential biomarkers}

Recent years, accompanied by the development of microarray techniques, miRNAs were systemically investigated in $\mathrm{PaC}$ patients. Wang et al. profiled four miRNAs, miR-21, miR-210, miR-155 and miR-196a as blood-based biomarkers of $\mathrm{PaC}$, with sensitivity of $64 \%$ and specificity of $89 \%[63,64]$. By comparing the miRNA expressions in $\mathrm{PaC}$ with normal pancreas/chronic pancreatitis in human tissue specimens and blood samples, Bauer et al. revealed that several miRNAs (miR-148a, miR-216a, miR-217, miR-181a/c, miR-324, miR-146a, miR-210, miR-345 and miR-574) were able to differentiate between $\mathrm{PaC}$ and normal/inflamed pancreas in tissue and blood specimens $[10,65]$. Notably, when they assessed miRNAs in blood samples only, they found that 36 miRNAs were able to distinguish $\mathrm{PaC}$ from healthy controls with sensitivity of $97.3 \%$ and specificity of $95 \%$. Liu et al. compared serum miRNA expressions of $\mathrm{PaC}$ patients with matched cancerfree controls and observed that seven miRNAs (miR-20a, miR-21, miR-24, miR-25, miR-99a, miR-185 and miR-191) were significantly altered in $\mathrm{PaC}$ patients $[12,66]$. 
Table 1 MiRNAs as early diagnostic markers for pancreatic caner

\begin{tabular}{|c|c|c|c|c|c|c|c|}
\hline \multirow[t]{2}{*}{ Biomarker } & \multirow[t]{2}{*}{ Source } & \multicolumn{3}{|c|}{ Sample size } & \multicolumn{2}{|c|}{ Diagnostic utility } & \multirow[t]{2}{*}{ Ref. } \\
\hline & & $\mathrm{PaC}$ & $\mathrm{CP}$ & Normal & Sensitivity & Specificity & \\
\hline miR-18a & plasma & 36 & & 30 & N/A & $\mathrm{N} / \mathrm{A}$ & [44] \\
\hline miR-192 & serum & 70 & & 40 & $76.00 \%$ & $55.00 \%$ & {$[46]$} \\
\hline miR-196a & serum & 35 & 15 & 15 & $100.00 \%$ & $75.00 \%$ & [48] \\
\hline miR-210 & plasma & 11 & & 11 & N/A & $\mathrm{N} / \mathrm{A}$ & [41] \\
\hline miR-221 & plasma & 47 & & 30 & $74.00 \%$ & $78.00 \%$ & {$[45]$} \\
\hline miR-1290 & serum & 41 & 35 & 19 & N/A & N/A & [52] \\
\hline miRNA-192,-194 & serum & N/A & & N/A & $84.00 \%$ & $75.00 \%$ & [47] \\
\hline miR-196a,b & serum & 24 & 10 & 10 & $100.00 \%$ & $90.00 \%$ & {$[50]$} \\
\hline miR-200a & serum & 45 & 11 & 32 & $84.40 \%$ & $87.50 \%$ & {$[42]$} \\
\hline miR-200b & serum & 45 & 11 & 32 & $71.10 \%$ & $96.90 \%$ & {$[42]$} \\
\hline miR-148a, $-216 a,-217,181 a / c,-324-5 p,-146 a,-210,-345,-574$ & blood & 45 & 38 & 33 & N/A & N/A & [61] \\
\hline miR-20a,-21,-24,-25,-99a,-185,-191 & serum & 95 & & 81 & $94.00 \%$ & $93.00 \%$ & [62] \\
\hline $\operatorname{miR}-21,-210,-155,-196 a$ & plasma & 28 & & 19 & $64.00 \%$ & $89.00 \%$ & [59] \\
\hline miR-22,-642,-885-5p & blood & 11 & & 11 & $91.00 \%$ & $91.00 \%$ & [64] \\
\hline CA $19-9$ and miR-27a-3p & serum/PBMCs & 129 & 103 & 60 & $85.30 \%$ & $81.60 \%$ & [66] \\
\hline CA $19-9$ and miR-16,-196a & serum/plasma & 140 & 111 & 68 & $92.00 \%$ & $95.60 \%$ & {$[67]$} \\
\hline CA $19-9$ and miR-145, $-150,-223,-636$ & serum/blood & 86 & 7 & 44 & $74.00 \%$ & $96.00 \%$ & [68] \\
\hline $\begin{array}{l}\text { CA } 19-9 \text { and miR-26b, }-34 a,-22,-126-5 p,-145,-150,-223 \text {, } \\
-505,-636,-885-5 p\end{array}$ & serum/blood & 86 & 7 & 44 & $73.00 \%$ & $97.00 \%$ & [68] \\
\hline
\end{tabular}

CA 19-9 Carbohydrate antigen 19-9, PaC Pancreatic cancer, CP Chronic pancreatitis

Ganepola et al. developed a panel of blood-based diagnostic biomarkers consisting of miR-642b, miR-885-5p and miR-22 for PaC early detection, with sensitivity of $91 \%$ and specificity of $91 \%[67,68]$. These findings indicate that combinations of miRNAs may proven to be more accurate at diagnosing and/or predicting outcome in $\mathrm{PaC}$ patients.

\section{Combination of panels of miRNAs with CA19-9 as biomarkers}

The idea of combining circulating miRNAs with CA19-9 for PaC early detection has also been raised up by some researchers. Wang et al. investigated miRNA expression levels in PBMCs in healthy, benign pancreatic/peripancreatic disease, and $\mathrm{PaC}$ cohorts. They found that the combination of miR-27a in PBMCs and serum CA19-9 levels showed higher diagnostic accuracy [69]. Liu et al. also elucidated the supplementary effect of plasma miRNAs with serum CA199 in early $\mathrm{PaC}$ diagnosis, and they demonstrated that miR-16 and miR-196a can discriminate $\mathrm{PaC}$ patients from healthy and $\mathrm{CP}$ patients. Moreover, the combination of miR-16, miR-196a and CA19-9 was more effective, with a sensitivity of $92 \%$ and specificity $95.6 \%$ for discriminating $\mathrm{PaC}$ patients from healthy controls, and a sensitivity of $88.4 \%$ and specificity of $96.3 \%$ for discriminating $\mathrm{PaC}$ from $\mathrm{CP}$ patients. Of note, the combination was sensitive in identifying in Stage $1 \mathrm{PaC}(85.2 \%)$ [70]. Schultz et al. have performed the largest screening of the whole blood miRNAs in $\mathrm{PaC}$ patients. In their study, the blood samples of $409 \mathrm{PaC}$ patients and 312 healthy participant and 25 patients with $\mathrm{CP}$ were tested. The test characteristics for the training cohort were index I (miR-145, miR-636, miR-223, and miR-150) and index II (miR-26b, miR-126-5p, miR-34a, miR-145, miR-22, miR-223, miR-150, miR-636, miR-505, and miR-885-5p). For CA19-9 and index I, the sensitivity was $74 \%$ and specificity was 96\% [71]. For CA19-9 and index II, the sensitivity was $73 \%$ and specificity was $97 \%$. Although most of the studies assessed circulating miRNAs in blood, study by Wang et al. investigated the miRNA profile in exocrine pancreatic secretions (pancreatic juice) from $\mathrm{PaC}$ patients [11]. Inclusion of serum CA19-9 with the profiles of four circulating miRNAs (miR-205, miR-210, miR-492, and miR-1427) was successfully discriminate pancreatic juice patients from $\mathrm{PaC}$ group, with a sensitivity to $91 \%$ and the specificity to $100 \%$ [72].

\section{Exosomal miRNAs as new promising biomarkers}

Exosomes, membrane vesicles ranging from 30 to $120 \mathrm{~nm}$ [73-76], are emerging as important intercellular communicators between tumor cells and their microenvionment via information transfer, including nucleotides and proteins [77-80]. Current clinical applications for exosomes in cancer are primarily early detection biomarkers and prognosis assessment. Because the relatively stable vesicle structure in the circulation, exosomes possess great potential for replacing or supplementing the currently used 
but unsatisfying biomarker CA19-9 [81-85]. Melo et al. showed that the expression of glypican-1 (GPC1) in $\mathrm{PaC}$-derived exosomes can be used in early $\mathrm{PaC}$ diagnosis $[86,87]$. In addition, Silva et al. reported that PDAC secreted exosomes induced liver metastatic burden, and could be served as a potential prognostic marker for detecting PDAC liver metastasis [77]. Compared with exosomal miRNAs that have been reported in several types of cancers, such as breast cancer and lung cancer, lesser studies focused on miRNAs in $\mathrm{PaC}$ secreted exosomes [84]. Que. et al. conducted a $\mathrm{PaC}$ exosome case-control study and found that exosomal miR-17-5p and miR-21 were enriched in the serum of $\mathrm{PaC}$ patients [88]. A study by Madhavan et al. showed that combination of a panel of proteins (CD44v6, Tspan8, EpCAM, MET and CD104) and four exosomal miRNAs (miR-1246, miR-4644, miR-3976 and miR-4306) markedly increased the diagnostic accuracy of $\mathrm{PaC}$ [89]. Recently, by exploring the new technology of label-free nanoplsmonic-based small noncoding RNA, Joshi et al. discovered that exosomal miR-10b was significantly increased in $\mathrm{PaC}$ patients $[84,90,91]$. Taken together, exosomal miRNAs show their potential as early detection and prognostic biomarkers in $\mathrm{PaC}$, however, larger numbers of extensive studies are necessary before the clinical application.

\section{Intracellular miRNAs: Potential therapeutic targets for chemoresistance of $\mathrm{PaC}$ \\ Molecular pathogenesis in $\mathrm{PaC}$ chemoresistance}

Another major barrier in successfully treating $\mathrm{PaC}$ is chemoresistance, which can cause the failure of the treatment and lead to high mortality of PaC. Statistic studies implicate that over $80 \%$ of the $\mathrm{PaC}$ patients showed local invasion or metastasis when diagnosed, which made them inoperable [92-94]. Thus, effective chemotherapy is extremely important for the treatment of advanced $\mathrm{PaC}$ patients. Of the numerous chemotherapeutic molecules that have been investigated, gemcitabine is used as the standard clinical drug used in $\mathrm{PaC}$ patients, and it usually combined with other adjuvant drugs in the treatment [94-99]. Although FLOFRINOX, another combination of four drugs has increased about 5 months survival than single gemcitabine treatment, this modest and incomplete benefit is unsatisfied, and there is still an urgent need for new drugs to combat chemoresistance in $\mathrm{PaC}$ patients [100].

The investigation of the underlying mechanisms of drug resistance has been lasted for half a century. It has been well-established that drug resistance could be either intrinsic (innate) or acquired during the treatment [101]. Conventionally, it is believed that the microenvironment surrounded tumor cells are responsible for innate drug resistance [102], while due to insensitivity to drug-induced apoptosis and the induction of drug-detoxification mechanisms, the drug resistance is acquired [103]. To date, a variety of distinct molecular mechanisms have been implicated to participate in $\mathrm{PaC}$ pathogenesis, including many genes, such as oncogenes and tumor suppressor genes [104-106], and several signaling pathways such as Notch, EGFR, Akt, NF- $k B$, TGF- $\beta$, JNK, and hedgehog [107-116]. These efforts trying to elucidate mechanisms of drug resistance prompted the development of new targeted agents. However, due to the highly complex nature of drug resistance, the current single agent or multiple drug combinations are often ineffective. The disappointing outcome demands a comprehensive understanding of drug-resistant mechanisms of $\mathrm{PaC}$. Recent studies have pointed out that the intracellular miRNAs may play important roles in cells with EMT-phenotype and cancer stem cells (CSCs) [117-119], making them as potential targets for reducing drug resistance.

\section{Role of intracellular miRNAs in PaC}

miRNAs are small RNAs that function as guide molecules in RNA silencing by base pairing with their target mRNAs, this posttranscriptional gene regulatory mechanism makes miRNAs as either "oncomiRs" or "tumor suppressors" [94, 120]. miRNA Profiling in PaC also has showed some of the aberrantly expressed miRNAs, including several miRNAs act as tumor suppressors, whereas others as oncomiRs [121-127]. Therefore, restoring the decreased tumor suppressor miRNAs enables reinstating "normal cellular programs" and hinders the "oncogenic progression". On the contrary, oncomiRs, are potential the therapeutic targets by RNA silencing. Notably, recent evidence suggests that miRNAs also play important roles in drug resistance, such as downregulation of miR-200 family is synonymous with gemcitabine-resistant $\mathrm{PaC}$ cells [128]. Meanwhile, tumor suppressor miR-145 directly targeted p70S6K1 and inhibited its expression, subsequently reverse the gemcitabine resistance [129]. Mikamori et al. revealed that long-term exposure of gemcitabine increase miR-155 expression in PDAC cell. These miR-155 not merely facilitated the anti-apoptotic activity in cells, but were also delivered by exosomes into other PDAC cells, therefore spreading the drug resistance widely [130].

\section{The EMT-like phenotype and miRNAs in PaC}

The epithelial cells can transformed from a cobblestone phenotype to a mesenchymal phenotype, which enables the epithelial cells to invade the extracellular matrix $[6,131]$. EMT was first described in early 1980s, but hasn't been paid enough attention until realizing it is closely related to tumor cell invasion and metastasis [132, 133]. When epithelial cells undergo an EMT process, the expression levels of E-cadherin 1 and junction plakoglobin are decreased, while the mesenchymal markers are increased, including vimentin, fibronectin and $\mathrm{N}$-cadherin $[134,135]$. Moreover, 
the activity of matrix metalloproteinases (MMPs) of epithelial cells were also increased, which contributes to the acquisition of invasiveness [134, 135]. Many studies have proven that EMT played an important role in chemoresistance [136, 137]. Arumugnam et al. reported that several pancreatic cell lines which showed high levels of epithelial markers were sensitive to chemotherapeutic drugs such as gemcitabine, whereas the cell lines resistant to these drugs showed the mesenchymal markers $[138,139]$.

Aberrant signaling pathways, cytokines and transcriptional factors contribute to the EMT process, thus targeting the EMT network could be a feasible approach to overcome chemoresistance $[94,140]$. Recent studies proven that the EMT process is regulated by different miRNAs, which function as critical regulators of the pathologic processes during cancer cell development [128] (Table 2). Philip et al. found that during the TGF- $\beta$-induced EMT process, miR-200 family and miR-205 were significantly decreased in cells [141, 142]. Overexpression of the miR-200 family could prevent TGF- $\beta$-stimulated cell EMT through inhibiting the expression levels of E-cadherin transcriptional repressors [143]. Following studies also confirmed that ectopic expression of miR-200 family played a determinant role in EMT. Restored miR-200 expression resulted in morphological reversal of EMT phenotype [128, 141, 144]. Sureban et al. illustrated the direct regulatory links between doublecortin-like kinase-1 (DCAMKL-1), miRNAs and EMT in $\mathrm{PaC}$ [145]. They found that knockdown of DCAMKL-1 induced miR-200a expression in human $\mathrm{PaC}$ cells, and consequently results in downregulation of EMT phenotypic transcription factors. Lzumchenko et al. demonstrated that the TGF $\beta$-miR-200-MIG6 network helps the EMT-kinase switch, which led to resistance to EGFR inhibitors [146]. Bao et al. found that activation of Notch-1signaling contributes to the switch of EMT phenotype through regulating miR-200b expression
[147]. Hamada et al. reported the tumor suppressor role of miR-126 in PaC cells by targeting disintegrin and ADM9 [148]. Recently, they also found that miR-197 stimulated EMT process in PaC cells by targeting p120 catenin [149]. Mody et al. found that histone methylation reversal agents, which were used to treat solid tumors, could attenuate TGF $\beta-1$ induced EMT features via restoring miR-663 and miR-4787-5p expression levels [150]. Other miRNAs have also been proven to participate in modulating cell EMT process. For example, let-7 was demonstrated to inhibit HMGA2 expression and maintain RAS-induced EMT [151-153]. Moes et al. found that the miR-203/SNAIL feedback loop regulates EMT process [154]. Ma et al. also reported that knockdown of miR-223 could attenuate drug resistance through reversing EMT phenotype [155].

\section{Cancer stem cells and miRNAs in PaC}

The CSC theory suggests that cancer cells can be divided into several different types of cells, including the large proportion of normal tumor cells and a small number of cancer stem cells (CSCs). However, these CSCs live longer, can generate new tumor cells, even cause relapse and distant metastasis [156]. This CSC theory explains why in many cancers, including $\mathrm{PaC}$, drugs that seem to rapidly reduce tumor size but failed to improve long-term survival [156-162]. Now it has been well established that CSCs are responsible for tumor initiation, propagation, and most importantly, they are chemoresistance, which cause the relapse of cancer [163].

Cell surface markers CD44, CD24, CD133, CXCR4 and ESA are expressed by $\mathrm{PaC}$ stem cells (approximately $1 \%$ of the tumor) $[117,163]$. Notably, even high doses of gemcitabine were unable to eliminate CSCs, although the majority of $\mathrm{PaC}$ cells were killed in the culture [117]. Studies suggest that the deregulated miRNAs may also contribute to pancreatic stem cells generation $[156,164,165]$ (Table 3).

Table 2 Deregulated miRNAs in pancreatic cancer and their functions in the EMT process

\begin{tabular}{|c|c|c|c|c|c|}
\hline \multirow[t]{2}{*}{ miRNAs } & \multirow[t]{2}{*}{ Targets } & \multirow[t]{2}{*}{ Status } & \multicolumn{2}{|l|}{ Functions in the EMT process } & \multirow[t]{2}{*}{ Ref. } \\
\hline & & & Activation & Inhitibion & \\
\hline miR-200a & ZEB1 & down & TGF- $\beta$ induced EMT Vimentin & P53 network E-cadherin & [126] \\
\hline miR-200b & ZEB2/SIP1 & & & & [188] \\
\hline miR-200c & Slug & & & & [189] \\
\hline miR-141 & SIRT & & & & [139] \\
\hline miR-429 & & & & & [142] \\
\hline miR-126 & ADM9 & down & Cellular migration invasion & E-cadherin & [146] \\
\hline miR-197 & P120 catenin & up & EMT process & & [147] \\
\hline miR-205 & ZEB2/SIP1 & down & & E-cadherin & [139] \\
\hline Let-7 & HMGA2 & down & RAS signaling & & {$[149,150]$} \\
\hline miR-217 & SIRT1 & down & TGF- $\beta$ induced EMT & & [151] \\
\hline miR-203 & SNAI1 & down & EMT process & & {$[152,154]$} \\
\hline
\end{tabular}


Table 3 Deregulated miRNAs and their functions in pancreatic cancer stem cells

\begin{tabular}{|c|c|c|c|c|c|}
\hline \multirow[t]{2}{*}{ miRNAs } & \multirow[t]{2}{*}{ Targets } & \multirow[t]{2}{*}{ Status } & \multicolumn{2}{|l|}{ Functions in CSCs } & \multirow[t]{2}{*}{ Ref. } \\
\hline & & & Activation & Inhibition & \\
\hline miR-200a & Suz12 & down & CD44 induction CSC formation CSC self-renewal & E-cadherin & [167] \\
\hline miR-200b & Bmi 1 & & & & [169] \\
\hline miR-200c & Notch 1 & & & & [168] \\
\hline miR-141 & $\operatorname{Lin} 28 B$ & & & & [166] \\
\hline miR-429 & FoxM1 & & & & {$[145,170,171,173$} \\
\hline miR-34a & $\mathrm{HDAC} 1 / 2$ & down & cell migration invasion & & [165] \\
\hline miR-101 & $\mathrm{EZH} 2 / \mathrm{MCL}-1 / \mathrm{Fos}$ & down & cell proliferation invasion & & {$[175]$} \\
\hline miR-125b & BAK1 & up & & apoptosis & [178] \\
\hline Let-7a & HMGA2 & down & & & [152] \\
\hline
\end{tabular}

CSCs Cancer stem cells

miR-34a was found to play a key role in $\mathrm{PaC}$ progression by inhibiting CSC characteristics, and restoration of miR-34a expression strongly inhibited the proliferation and invasion of $\mathrm{PaC}$ CSCs [166]. Wu et al. demonstrated that miR-34 regulates drug resistance via targeting HDAC1 and HDAC2 [167]. Bao et al. showed that metformin could decrease the CSC marker expression via reversal of miRNAs that are significantly decreased in $\mathrm{PaC}$, such as let 7 and miR-200 family [168]. Here, miR-200 family has also been shown to inhibit Suz12 and Bmil, two essential genes responsible for the stem cell maintenance [144, 169-171]. Notch signaling pathway has been demonstrated to be key regulator in the CSC formation. MiR-200b could repress Lin28b and Notch 1 to inhibit the CSC proliferation and up-regulate CD44 expression [147, 172-174]. Bao et al. demonstrated that overexpression of miR-200b also inhibited the FoxM1 and increased cell migration $[175,176]$. By studying the tumor suppressor miR-101, Konno et al. reported that miR-101/EZH2/MCL-1/Fos axis induces the apoptosis and senescence of cancer cell [177]. Yang et al. found that let-7a increase the drug sensitivity via downregulate HMGA2 [178]. Jung et al. showed several miRNAs were significantly altered in pancreatic CSCs, such as miR-99a, miR-100 etc. [179]. Inhibition of apoptosis via downregulating BAK1 was reported recently by Chen and co-workers [180]. Recently, Haseqawa et al. reported that miR-1246 contributed to drug resistance and CSC properties in $\mathrm{PaC}$, furthermore, it could be promising prognosis marker for $\mathrm{PaC}$ patients [181].

\section{Targeting specific miRNAs to reduce chemoresistance}

Accumulating evidences suggest the pivotal roles of EMT-type cells and CSCs in drug resistance, as miRNAs appear to exert ubiquitous regulatory roles in EMT and CSCs, inhibiting or restoring the deregulated miRNAs could become a novel approach for $\mathrm{PaC}$ treatment by eliminating of CSCs or EMT-like cells [6]. For example, inhibition of the aberrantly expressed miR-221 and
miR-21 significantly improved gemcitabine sensitivity in $\mathrm{PaC}$ cells [122, 182], whereas introduction of miR-200 family could make the gemcitabine-resistance cells become sensitive to gemcitabine again [121, 183, 184]. Some researchers reported that natural agents isolated from common food, such as curcumin, isoflavone and idole-3-carbinol, could reverse EMT-phenotype by regulating miRNAs, which could be an easy and safe way for treating $\mathrm{PaC}$ patients $[185,186]$.

\section{Conclusion and future perspective}

Intracellular miRNAs are closely correlated with the pathogenesis of PaC. Targeting specific miRNAs become new potential strategy to treat $\mathrm{PaC}$ and reduce drug-resistance. In addition, in the recent decade, the detection of stably expressed circulating miRNAs in blood is a 'booming' field in biomarker world [68]. The high stability of miRNAs in circulation postulate the possibility of using them as sensitive and specific biomarkers for $\mathrm{PaC}$ early diagnosis and prognosis, from single miRNA to a panel of miRNAs profiling, and in certain cases, from miRNA expression profile alone to a combination of miRNA profile with other clinic $\mathrm{PaC}$ indicators such as CA19-9. However, although these findings have the potential to improve clinical early diagnosis in the future, it is fair to say that all these potential biomarkers still need rigorous validation before using in routine management. Circulating miRNAs also show the implication of chemoresistance in several cancers such as breast cancer [187]. Several circulating miRNAs present functional significance in predicting the resistance to chemotherapy $[188,189]$. Thus, it is reasonable to further explore the role of circulating miRNAs in the development of chemoresistance in $\mathrm{PaC}$. Despite the promising therapeutic advantages of miRNAs, there are several critical issues, such as avoiding "off-target" effects, optimizing the miRNA dosing and devising effective delivery approaches, need to be fully addressed before it can benefit $\mathrm{PaC}$ patients. 


\section{Abbreviations}

ADM9: Metalloproteinase domain-containing protein 9; Akt-2: v-akt murine thymoma viral oncogene homolog 2; BAK1: B-cell lymphoma 2-antagonist/ killer 1; BCl-6: B-cell lymphoma 6; CA19-9: Carbohydrate antigen 19-9; CCNG2: Cyclin G2; CSC: Cancer stem cell; DCAMKL-1: Deouble ecortin-like kinase-1; EGFR: Epidermal growth factor receptor; EMT: Epithelial-mesenchymal transition; ESA: Epithelial-specific antigen; Hh: Hedgehog; HMG2: High-mobility group protein 2; JNK: c-Jun N-terminal kinases; K-ras: V-Ki-ras2 Kirsten rat sarcoma viral oncogene homolog; MIA: Melanoma inhibitory activity; MIG6: Mitogen-inducible gene 6; MMP: Metalloproteinase; Myb: Myelobastosis oncogene; NF-kB: Nuclear factor-k-gene binding; P16: Cyclin-dependent kinase inhibitor 2A; P53: Tumor protein 53; PaC: Pancreatic cancer; PBMC: Peripheral blood mononuclear cell; PRC: Polycomb repressor complexe; Pten: Phosphatase and tensin homolog; S100P: S100 calcium binding protein P; Slug: Snail homolog 2; Smad4: Mothers against DPP homolog 4; Src: Sarcoma oncogene; TGF- $\beta$ : Transforming growth factor; ZEB: Zinc finger E-box binding homeobox

\section{Acknowledgments}

Not applicable.

\section{Funding}

This work was supported by grants from the National Basic Research Program of China (973 Program, 2012CB517603 and 2011CB504803), the National Key Technology R\&D Program (No. 2011BAl10B04).

\section{Availability of data and materials}

Not applicable.

\section{Authors' contributions}

$\mathrm{XJ}, \mathrm{J}$ and $\mathrm{DH}$ wrote the manuscript. ZW and $\mathrm{SZ}$ contributed to the discussion. $X J, J L$ and $Y Z$ revised the manuscript and approved the final version to be published. All authors read and approved the final manuscript.

\section{Ethics approval and consent to participate}

Not applicable.

\section{Consent for publication}

Not applicable.

\section{Competing interests}

The authors declare that they have no competing interests.

\section{Publisher's Note}

Springer Nature remains neutral with regard to jurisdictional claims in published maps and institutional affiliations.

Received: 24 May 2017 Accepted: 27 February 2019

Published online: 03 April 2019

\section{References}

1. Ilic M, llic I. Epidemiology of pancreatic cancer. World J Gastroenterol. 2016;22(44):9694-705.

2. Smith RA, et al. Cancer screening in the United States, 2016: a review of current American Cancer Society guidelines and current issues in cancer screening. CA Cancer J Clin. 2016;66(2):96-114.

3. Bao B, et al. Overview of cancer stem cells (CSCs) and mechanisms of their regulation: implications for cancer therapy. Curr Protoc Pharmacol. 2013; Chapter 14: p. Unit 1425.

4. Jemal A, et al. Cancer statistics, 2010. CA Cancer J Clin. 2010:60(5):277-300.

5. Vincent A, et al. Pancreatic cancer. Lancet. 2011;378(9791):607-20.

6. Wang Z, et al. Pancreatic cancer: understanding and overcoming chemoresistance. Nat Rev Gastroenterol Hepatol. 2011;8(1):27-33.

7. Costello E, Greenhalf W, Neoptolemos JP. New biomarkers and targets in pancreatic cancer and their application to treatment. Nat Rev Gastroenterol Hepatol. 2012;9(8):435-44.

8. Lagos-Quintana $\mathrm{M}$, et al. Identification of novel genes coding for small expressed RNAs. Science. 2001;294(5543):853-8.

9. Mardin WA, Mees ST. MicroRNAs: novel diagnostic and therapeutic tools for pancreatic ductal adenocarcinoma. Ann Surg Oncol. 2009;16(11):3183-9.

10. Cortez MA, et al. MicroRNAs in body fluids--the mix of hormones and biomarkers. Nat Rev Clin Oncol. 2011;8(8):467-77.
11. Albulescu R, et al. Tissular and soluble miRNAs for diagnostic and therapy improvement in digestive tract cancers. Expert Rev Mol Diagn. 2011;11(1):101-20.

12. Liu $R$, et al. Serum microRNA expression profile as a biomarker in the diagnosis and prognosis of pancreatic cancer. Clin Chem. 2012;58(3):610-8.

13. Fassan $\mathrm{M}$, et al. Early miR-223 upregulation in gastroesophageal carcinogenesis. Am J Clin Pathol. 2017:147(3):301-8.

14. Shaker O, et al. miRNA-101-1 and miRNA-221 expressions and their polymorphisms as biomarkers for early diagnosis of hepatocellular carcinoma. Infect Genet Evol. 2017;51:173-81.

15. Hawa Z, et al. The miRacle in pancreatic Cancer by miRNAs: tiny angels or devils in disease progression. Int J Mol Sci. 2016;17(6). https://doi.org/10. 3390/ijms 17060809.

16. Chakraborty $\mathrm{S}$, et al. Current status of molecular markers for early detection of sporadic pancreatic cancer. Biochim Biophys Acta. 2011;1815(1):44-64.

17. Azmi AS, et al. Aberrant epigenetic grooming of miRNAs in pancreatic cancer: a systems biology perspective. Epigenomics. 2011;3(6):747-59.

18. Neoptolemos JP, et al. A randomized trial of chemoradiotherapy and chemotherapy after resection of pancreatic cancer. $N$ Engl J Med. 2004:350(12):1200-10.

19. Neoptolemos JP, et al. Adjuvant 5-fluorouracil and folinic acid vs observation for pancreatic cancer: composite data from the ESPAC-1 and $-3(\mathrm{v} 1)$ trials. Br J Cancer. 2009;100(2):246-50.

20. Neoptolemos JP, et al. Adjuvant chemotherapy with fluorouracil plus folinic acid vs gemcitabine following pancreatic cancer resection: a randomized controlled trial. JAMA. 2010;304(10):1073-81.

21. Kuvshinoff BW, Bryer MP. Treatment of resectable and locally advanced pancreatic cancer. Cancer Control. 2000;7(5):428-36.

22. Holly EA, et al. Signs and symptoms of pancreatic cancer: a populationbased case-control study in the San Francisco Bay area. Clin Gastroenterol Hepatol. 2004;2(6):510-7.

23. Sun T, et al. Aberrant MicroRNAs in pancreatic Cancer: researches and clinical implications. Gastroenterol Res Pract. 2014;2014:386561.

24. Xu Q, Zhang TP, Zhao YP. Advances in early diagnosis and therapy of pancreatic cancer. Hepatobiliary Pancreat Dis Int. 2011;10(2):128-35.

25. Ballehaninna UK, Chamberlain RS. Biomarkers for pancreatic cancer: promising new markers and options beyond CA 19-9. Tumour Biol. 2013;34(6):3279-92.

26. Kilic M, et al. Value of preoperative serum CA $19-9$ levels in predicting resectability for pancreatic cancer. Can J Surg. 2006;49(4):241-4.

27. Ballehaninna UK, Chamberlain RS. The clinical utility of serum CA 19-9 in the diagnosis, prognosis and management of pancreatic adenocarcinoma: an evidence based appraisal. J Gastrointest Oncol. 2012;3(2):105-19.

28. Harsha $\mathrm{HC}$, et al. A compendium of potential biomarkers of pancreatic cancer. PLoS Med. 2009;6(4):e1000046.

29. Duffy MJ, et al. Tumor markers in pancreatic cancer: a European group on tumor markers (EGTM) status report. Ann Oncol. 2010;21(3):441-7.

30. Tanase CP, et al. Advances in pancreatic cancer detection. Adv Clin Chem. 2010;51:145-80

31. Ballehaninna UK, Chamberlain RS. Serum CA 19-9 as a biomarker for pancreatic Cancer-a comprehensive review. Indian J Surg Oncol. 2011;2(2):88-100.

32. Bunger $\mathrm{S}$, et al. Serum biomarkers for improved diagnostic of pancreatic cancer: a current overview. J Cancer Res Clin Oncol. 2011;137(3):375-89.

33. Kosaka N, lguchi H, Ochiya T. Circulating microRNA in body fluid: a new potential biomarker for cancer diagnosis and prognosis. Cancer Sci. 2010; 101(10):2087-92.

34. Lu J, et al. MicroRNA expression profiles classify human cancers. Nature. 2005;435(7043):834-8.

35. Waldman SA, Terzic A. MicroRNA signatures as diagnostic and therapeutic targets. Clin Chem. 2008;54(6):943-4.

36. Lothe L, Lindberg T, Jakobsson I. Macromolecular absorption in infants with infantile colic. Acta Paediatr Scand. 1990;79(4):417-21.

37. Gao W, Xu J, Shu YQ. miRNA expression and its clinical implications for the prevention and diagnosis of non-small-cell lung cancer. Expert Rev Respir Med. 2011:5(5):699-709.

38. Chen $X$, et al. Characterization of microRNAs in serum: a novel class of biomarkers for diagnosis of cancer and other diseases. Cell Res. 2008;18(10):997-1006.

39. Huang Z, et al. Plasma microRNAs are promising novel biomarkers for early detection of colorectal cancer. Int J Cancer. 2010;127(1):118-26.

40. Arroyo JD, et al. Argonaute2 complexes carry a population of circulating microRNAs independent of vesicles in human plasma. Proc Natl Acad Sci U S A. 2011;108(12):5003-8. 
41. Vickers $\mathrm{KC}$, et al. MicroRNAs are transported in plasma and delivered to recipient cells by high-density lipoproteins. Nat Cell Biol. 2011;13(4):423-33.

42. Turchinovich A, et al. Characterization of extracellular circulating microRNA. Nucleic Acids Res. 2011;39(16):7223-33.

43. Kosaka N, et al. Secretory mechanisms and intercellular transfer of microRNAs in living cells. J Biol Chem. 2010;285(23):17442-52.

44. Zhang $Y$, et al. Secreted monocytic miR-150 enhances targeted endothelial cell migration. Mol Cell. 2010;39(1):133-44.

45. Ho AS, et al. Circulating miR-210 as a novel hypoxia marker in pancreatic Cancer. Transl Oncol. 2010;3(2):109-13.

46. Li A, et al. Pancreatic cancers epigenetically silence SIP1 and hypomethylate and overexpress miR-200a/200b in association with elevated circulating miR-200a and miR-200b levels. Cancer Res. 2010;70(13):5226-37.

47. Komatsu S, et al. Circulating miR-18a: a sensitive cancer screening biomarker in human cancer. In Vivo. 2014;28(3):293-7.

48. Morimura $\mathrm{R}$, et al. Novel diagnostic value of circulating miR-18a in plasma of patients with pancreatic cancer. Br J Cancer. 2011;105(11):1733-40.

49. Kawaguchi $T$, et al. Clinical impact of circulating miR-221 in plasma of patients with pancreatic cancer. Br J Cancer. 2013;108(2):361-9.

50. Zhao C, et al. Diagnostic and biological significance of microRNA-192 in pancreatic ductal adenocarcinoma. Oncol Rep. 2013;30(1):276-84.

51. Zhang J, et al. Upregulation of miR-194 contributes to tumor growth and progression in pancreatic ductal adenocarcinoma. Oncol Rep. 2014;31(3): 1157-64.

52. Kong $X$, et al. Detection of differentially expressed microRNAs in serum of pancreatic ductal adenocarcinoma patients: miR-196a could be a potentia marker for poor prognosis. Dig Dis Sci. 2011;56(2):602-9.

53. Frampton $A E$, et al. microRNAs as markers of survival and chemoresistance in pancreatic ductal adenocarcinoma. Expert Rev Anticancer Ther. 2011; 11(12):1837-42.

54. Slater EP, et al. MicroRNA-196a and -196b as potential biomarkers for the early detection of familial pancreatic Cancer. Transl Oncol. 2014;7(4):464-71.

55. Sun $B$, et al. Downregulation of miR-124 predicts poor prognosis in pancreatic ductal adenocarcinoma patients. Br J Biomed Sci. 2016;73(4):152-7.

56. Li A, et al. MicroRNA array analysis finds elevated serum miR-1290 accurately distinguishes patients with low-stage pancreatic cancer from healthy and disease controls. Clin Cancer Res. 2013;19(13):3600-10.

57. Le Large $T Y$, et al. Circulating microRNAs as diagnostic biomarkers for pancreatic cancer. Expert Rev Mol Diagn. 2015;15(12):1525-9.

58. Safavi M, Sabourian R, Abdollahi M. The development of biomarkers to reduce attrition rate in drug discovery focused on oncology and central nervous system. Expert Opin Drug Discov. 2016;11(10):939-56.

59. Hernandez YG, Lucas AL. MicroRNA in pancreatic ductal adenocarcinoma and its precursor lesions. World J Gastrointest Oncol. 2016;8(1):18-29.

60. Deng $T$, et al. Identification of circulating MiR-25 as a potential biomarker for pancreatic Cancer diagnosis. Cell Physiol Biochem. 2016;39(5):1716-22.

61. Alemar B, et al. miRNA-21 and miRNA-34a are potential minimally invasive biomarkers for the diagnosis of pancreatic ductal adenocarcinoma. Pancreas. 2016:45(1):84-92.

62. Chen Q, et al. Circulating microRNA-182 in plasma and its potential diagnostic and prognostic value for pancreatic cancer. Med Oncol. 2014;31(11):225

63. Wang J, et al. MicroRNAs in plasma of pancreatic ductal adenocarcinoma patients as novel blood-based biomarkers of disease. Cancer Prev Res (Phila). 2009:2(9):807-13.

64. Vychytilova-Faltejskova P, Slaby O. Circulating blood-borne microRNAs as biomarkers in solid tumors. EXS. 2015;106:75-122.

65. Bauer AS, et al. Diagnosis of pancreatic ductal adenocarcinoma and chronic pancreatitis by measurement of microRNA abundance in blood and tissue. PLoS One. 2012;7(4):e34151.

66. Visani $M$, et al. Contribution of microRNA analysis to characterisation of pancreatic lesions: a review. J Clin Pathol. 2015;68(11):859-69.

67. Ganepola GA, et al. Novel blood-based microRNA biomarker panel for early diagnosis of pancreatic cancer. World J Gastrointest Oncol. 2014;6(1):22-33.

68. Zen K, Zhang CY. Circulating microRNAs: a novel class of biomarkers to diagnose and monitor human cancers. Med Res Rev. 2012;32(2):326-48.

69. Wang WS, et al. Combined serum CA19-9 and miR-27a-3p in peripheral blood mononuclear cells to diagnose pancreatic cancer. Cancer Prev Res (Phila). 2013;6(4):331-8.

70. Liu J, et al. Combination of plasma microRNAs with serum CA19-9 for early detection of pancreatic cancer. Int J Cancer. 2012;131(3):683-91.
71. Schultz NA, et al. MicroRNA biomarkers in whole blood for detection of pancreatic cancer. JAMA. 2014;311(4):392-404.

72. Wang J, et al. Circulating microRNAs in pancreatic juice as candidate biomarkers of pancreatic Cancer. J Cancer. 2014:5(8):696-705.

73. Arscott WT, Camphausen KA. EGFR isoforms in exosomes as a novel method for biomarker discovery in pancreatic cancer. Biomark Med. 2011;5(6):821.

74. Record $M$, et al. Exosomes as new vesicular lipid transporters involved in cell-cell communication and various pathophysiologies. Biochim Biophys Acta. 2014;1841(1):108-20.

75. S ELA, et al. Extracellular vesicles: biology and emerging therapeutic opportunities. Nat Rev Drug Discov. 2013;12(5):347-57.

76. Costa-Silva B, et al. Pancreatic cancer exosomes initiate pre-metastatic niche formation in the liver. Nat Cell Biol. 2015;17(6):816-26.

77. Matsushita $\mathrm{H}$, et al. Exosome migration inhibitory factor as a marker and therapeutic target for pancreatic Cancer. Gastroenterology. 2016;150(4): 1033-5.

78. Jin $\mathrm{H}$, Wu Y, Tan $X$. The role of pancreatic cancer-derived exosomes in cancer progress and their potential application as biomarkers. Clin Transl Oncol. 2017:19(8):921-30

79. Nuzhat $Z$, et al. Tumour-derived exosomes as a signature of pancreatic cancer - liquid biopsies as indicators of tumour progression. Oncotarget. 2017;8(10):17279-91.

80. Falasca M, Kim M, Casari I. Pancreatic cancer: current research and future directions. Biochim Biophys Acta. 2016;1865(2):123-32.

81. Vader P, Breakefield XO, Wood MJ. Extracellular vesicles: emerging targets for cancer therapy. Trends Mol Med. 2014;20(7):385-93.

82. Erb U, Zoller M. Progress and potential of exosome analysis for early pancreatic cancer detection. Expert Rev Mol Diagn. 2016;16(7):757-67.

83. Patel GK, et al. Pancreatic Cancer exosomes: shedding off for a meaningful journey. Pancreat Disord Ther. 2016;6(2):e148.

84. Lu L, Risch HA. Exosomes: potential for early detection in pancreatic cancer. Future Oncol. 2016;12(8):1081-90.

85. Babic A, Wolpin BM. Circulating exosomes in pancreatic Cancer: will they succeed on the long, littered road to early detection marker? Clin Chem. 2016;62(2):307-9.

86. Weber C. Biomarkers: the challenge to find biomarkers for the early detection of pancreatic cancer. Nat Rev Gastroenterol Hepatol. 2015;12(8):427.

87. Thery C. Cancer: diagnosis by extracellular vesicles. Nature. 2015:523(7559): $161-2$.

88. Que R, et al. Analysis of serum exosomal microRNAs and clinicopathologic features of patients with pancreatic adenocarcinoma. World J Surg Oncol. 2013;11:219.

89. Madhavan B, et al. Combined evaluation of a panel of protein and miRNA serum-exosome biomarkers for pancreatic cancer diagnosis increases sensitivity and specificity. Int J Cancer. 2015;136(11):2616-27.

90. Joshi GK, et al. Label-free Nanoplasmonic-based short noncoding RNA sensing at Attomolar concentrations allows for quantitative and highly specific assay of MicroRNA-10b in biological fluids and circulating exosomes. ACS Nano. 2015:9(11):11075-89.

91. Foroni $C$, et al. Epithelial-mesenchymal transition and breast cancer: role, molecular mechanisms and clinical impact. Cancer Treat Rev. 2012;38(6):689-97.

92. Siegel R, et al. Cancer statistics, 2014. CA Cancer J Clin. 2014;64(1):9-29.

93. Matsuno $\mathrm{S}$, et al. Pancreatic Cancer registry in Japan: 20 years of experience. Pancreas. 2004;28(3):219-30.

94. Chitkara D, Mittal A, Mahato RI. miRNAs in pancreatic cancer: therapeutic potential, delivery challenges and strategies. Adv Drug Deliv Rev. 2015;81: 34-52.

95. Von Hoff DD, et al. Increased survival in pancreatic cancer with nabpaclitaxel plus gemcitabine. N Engl J Med. 2013;369(18):1691-703.

96. Portal $\mathrm{A}$, et al. Sustained response with gemcitabine plus nab-paclitaxel after folfirinox failure in metastatic pancreatic cancer: report of an effective new strategy. Clin Res Hepatol Gastroenterol. 2014;38(2):e23-6.

97. Park $\mathrm{S}$, et al. Phase II trial of Erlotinib plus gemcitabine chemotherapy in Korean patients with advanced pancreatic Cancer and prognostic factors for chemotherapeutic response. Gut Liver. 2013;7(5):611-5.

98. Affeld $\mathrm{K}$, et al. How can we achieve infection-resistant percutaneous energy transfer? Artif Organs. 2011;35(8):800-6.

99. Costello BA, et al. Phase I trial of everolimus, gemcitabine and cisplatin in patients with solid tumors. Investig New Drugs. 2014;32(4):710-6.

100. Conroy T, et al. FOLFIRINOX versus gemcitabine for metastatic pancreatic cancer. N Engl J Med. 2011;364(19):1817-25. 
101. Szakacs G, et al. Targeting multidrug resistance in cancer. Nat Rev Drug Discov. 2006;5(3):219-34.

102. Damiano JS. Integrins as novel drug targets for overcoming innate drug resistance. Curr Cancer Drug Targets. 2002;2(1):37-43.

103. Gottesman MM. Mechanisms of cancer drug resistance. Annu Rev Med. 2002:53:615-27.

104. Schutte $M$, et al. Abrogation of the Rb/p16 tumor-suppressive pathway in virtually all pancreatic carcinomas. Cancer Res. 1997;57(15):3126-30.

105. Li Y, et al. Molecular analysis of the p53 gene in pancreatic adenocarcinoma. Diagn Mol Pathol. 1998;7(1):4-9.

106. Cowgill SM, Muscarella P. The genetics of pancreatic cancer. Am J Surg. 2003;186(3):279-86.

107. Almoguera $C$, et al. Most human carcinomas of the exocrine pancreas contain mutant c-K-ras genes. Cell. 1988;53(4):549-54.

108. Sarkar FH, Banerjee S, Li Y. Pancreatic cancer: pathogenesis, prevention and treatment. Toxicol Appl Pharmacol. 2007;224(3):326-36.

109. Scaltriti M, Baselga J. The epidermal growth factor receptor pathway: a model for targeted therapy. Clin Cancer Res. 2006;12(18):5268-72.

110. Chadha KS, et al. Activated Akt and Erk expression and survival after surgery in pancreatic carcinoma. Ann Surg Oncol. 2006;13(7):933-9.

111. Yang SH, et al. Nuclear expression of glioma-associated oncogene homolog 1 and nuclear factor-kappaB is associated with a poor prognosis of pancreatic cancer. Oncology. 2013;85(2):86-94.

112. Algul H, Adler G, Schmid RM. NF-kappaB/Rel transcriptional pathway: implications in pancreatic cancer. Int J Gastrointest Cancer. 2002;31(1-3):71-8

113. Sclabas GM, et al. NF-kappaB in pancreatic cancer. Int J Gastrointest Cancer. 2003;33(1):15-26

114. Maniati $\mathrm{E}_{\text {, et }}$ al. Crosstalk between the canonical NF-kappaB and notch signaling pathways inhibits Ppargamma expression and promotes pancreatic cancer progression in mice. J Clin Invest. 2011;121(12):4685-99.

115. Thayer SP, et al. Hedgehog is an early and late mediator of pancreatic cancer tumorigenesis. Nature. 2003;425(6960):851-6.

116. Perdigao-Henriques $\mathrm{R}$, et al. miR-200 promotes the mesenchymal to epithelial transition by suppressing multiple members of the Zeb2 and Snail1 transcriptional repressor complexes. Oncogene. 2016;35(2):158-72.

117. Hermann PC, et al. Distinct populations of cancer stem cells determine tumor growth and metastatic activity in human pancreatic cancer. Cell Stem Cell. 2007;1(3):313-23.

118. Voulgari A, Pintzas A. Epithelial-mesenchymal transition in cancer metastasis: mechanisms, markers and strategies to overcome drug resistance in the clinic. Biochim Biophys Acta. 2009;1796(2):75-90.

119. Sarkar $\mathrm{FH}$, et al. Implication of microRNAs in drug resistance for designing novel cancer therapy. Drug Resist Updat. 2010;13(3):57-66.

120. Costa PM, Pedroso de Lima MC. MicroRNAs as molecular targets for Cancer therapy: on the modulation of MicroRNA expression. Pharmaceuticals (Basel). 2013;6(10):1195-220.

121. Moriyama T, et al. MicroRNA-21 modulates biological functions of pancreatic cancer cells including their proliferation, invasion, and chemoresistance. Mol Cancer Ther. 2009:8(5):1067-74.

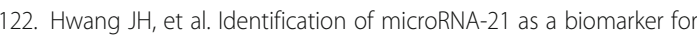
chemoresistance and clinical outcome following adjuvant therapy in resectable pancreatic cancer. PLoS One. 2010;5(5):e10630.

123. Nakata K, et al. MicroRNA-10b is overexpressed in pancreatic cancer, promotes its invasiveness, and correlates with a poor prognosis. Surgery. 2011;150(5):916-22.

124. Zhu Z, et al. Expression of microRNA-218 in human pancreatic ductal adenocarcinoma and its correlation with tumor progression and patient survival. J Surg Oncol. 2014;109(2):89-94.

125. Singh $\mathrm{S}$, et al. miRNA profiling in pancreatic cancer and restoration of chemosensitivity. Cancer Lett. 2013;334(2):211-20.

126. Mittal A, et al. Efficacy of gemcitabine conjugated and miRNA-205 complexed micelles for treatment of advanced pancreatic cancer Biomaterials. 2014;35(25):7077-87.

127. Cai $B$, et al. miRNA-181b increases the sensitivity of pancreatic ductal adenocarcinoma cells to gemcitabine in vitro and in nude mice by targeting BCL-2. Oncol Rep. 2013;29(5):1769-76.

128. Li Y, et al. Up-regulation of miR-200 and let-7 by natural agents leads to the reversal of epithelial-to-mesenchymal transition in gemcitabine-resistant pancreatic cancer cells. Cancer Res. 2009;69(16):6704-12.

129. Lin $Y$, et al. MiRNA-145 increases therapeutic sensibility to gemcitabine treatment of pancreatic adenocarcinoma cells. Oncotarget. 2016;7(43): 70857-68.
130. Mikamori M, et al. MicroRNA-155 controls exosome synthesis and promotes gemcitabine resistance in pancreatic ductal adenocarcinoma. Sci Rep. 2017;7:42339.

131. Mjaatvedt CH, Markwald RR. Induction of an epithelial-mesenchymal transition by an in vivo adheron-like complex. Dev Biol. 1989;136(1):118-28.

132. Thompson EW, et al. Oncogene-induced basement membrane invasiveness in human mammary epithelial cells. Clin Exp Metastasis. 1994;12(3):181-94.

133. Savagner $P$, et al. Modulations of the epithelial phenotype during embryogenesis and cancer progression. Cancer Treat Res. 1994;71:229-49.

134. Thiery JP, et al. Epithelial-mesenchymal transitions in development and disease. Cell. 2009;139(5):871-90.

135. Lou $C$, et al. Naringenin decreases invasiveness and metastasis by inhibiting TGF-beta-induced epithelial to mesenchymal transition in pancreatic cancer cells. PLoS One. 2012;7(12):e50956.

136. Izumiya $M$, et al. Chemoresistance is associated with cancer stem cell-like properties and epithelial-to-mesenchymal transition in pancreatic cancer cells. Anticancer Res. 2012:32(9):3847-53.

137. Wang Z, et al. Acquisition of epithelial-mesenchymal transition phenotype of gemcitabine-resistant pancreatic cancer cells is linked with activation of the notch signaling pathway. Cancer Res. 2009;69(6):2400-7.

138. Arumugam $T$, et al. Epithelial to mesenchymal transition contributes to drug resistance in pancreatic cancer. Cancer Res. 2009;69(14):5820-8.

139. Munoz $P$, lliou MS, Esteller M. Epigenetic alterations involved in cancer stem cell reprogramming. Mol Oncol. 2012;6(6):620-36.

140. Castellanos JA, Merchant NB, Nagathihalli NS. Emerging targets in pancreatic cancer: epithelial-mesenchymal transition and cancer stem cells. Onco Targets Ther. 2013;6:1261-7.

141. Gregory PA, et al. The miR-200 family and miR-205 regulate epithelial to mesenchymal transition by targeting ZEB1 and SIP1. Nat Cell Biol. 2008;10(5):593-601.

142. Nothnick WB. The role of micro-RNAs in the female reproductive tract Reproduction. 2012;143(5):559-76.

143. Sun $L$, et al. MiR-200b and miR-15b regulate chemotherapy-induced epithelial-mesenchymal transition in human tongue cancer cells by targeting BMI1. Oncogene. 2012;31(4):432-45.

144. Feng B, Wang R, Chen LB. Review of miR-200b and cancer chemosensitivity. Biomed Pharmacother. 2012;66(6):397-402.

145. Sureban SM, et al. DCAMKL-1 regulates epithelial-mesenchymal transition in human pancreatic cells through a miR-200a-dependent mechanism. Cancer Res. 2011;71(6):2328-38.

146. Izumchenko E, et al. The TGFbeta-miR200-MIG6 pathway orchestrates the EMT-associated kinase switch that induces resistance to EGFR inhibitors. Cancer Res. 2014;74(14):3995-4005

147. Bao B, et al. Notch-1 induces epithelial-mesenchymal transition consistent with cancer stem cell phenotype in pancreatic cancer cells. Cancer Lett. 2011;307(1):26-36.

148. Hamada S, et al. MiR-126 acts as a tumor suppressor in pancreatic cancer cells via the regulation of ADAM9. Mol Cancer Res. 2012;10(1):3-10.

149. Hamada S, et al. miR-197 induces epithelial-mesenchymal transition in pancreatic cancer cells by targeting p120 catenin. J Cell Physiol. 2013;228(6):1255-63.

150. Mody HR, et al. Inhibition of S-Adenosylmethionine-dependent methyltransferase attenuates TGFbeta1-induced EMT and metastasis in pancreatic Cancer: putative roles of miR-663a and miR-4787-5p. Mol Cancer Res. 2016;14(11):1124-35.

151. Watanabe $\mathrm{S}$, et al. HMGA2 maintains oncogenic RAS-induced epithelialmesenchymal transition in human pancreatic cancer cells. Am J Pathol. 2009;174(3):854-68.

152. Liu Q, et al. HMGA2 is down-regulated by microRNA let-7 and associated with epithelial-mesenchymal transition in oesophageal squamous cell carcinomas of Kazakhs. Histopathology. 2014;65(3):408-17.

153. Zhao G, et al. Chronic pancreatitis and pancreatic cancer demonstrate active epithelial-mesenchymal transition profile, regulated by miR-217-SIRT1 pathway. Cancer Lett. 2014;355(2):184-91.

154. Moes $M$, et al. A novel network integrating a miRNA-203/SNAl1 feedback loop which regulates epithelial to mesenchymal transition. PLoS One. 2012;7(4):e35440.

155. Ma J, et al. Synergistic reversal effect of epithelial-to-mesenchymal transition by miR-223 inhibitor and genistein in gemcitabine-resistant pancreatic cancer cells. Am J Cancer Res. 2016;6(6):1384-95.

156. Delude C. Tumorigenesis: testing ground for cancer stem cells. Nature. 2011;480(7377):S43-5 
157. Eppert K, et al. Stem cell gene expression programs influence clinical outcome in human leukemia. Nat Med. 2011;17(9):1086-93.

158. Tang C, Ang BT, Pervaiz S. Cancer stem cell: target for anti-cancer therapy. FASEB J. 2007;21(14):3777-85

159. Ma S, et al. Identification and characterization of tumorigenic liver cancer stem/progenitor cells. Gastroenterology. 2007;132(7):2542-56.

160. O'Brien CA, et al. A human colon cancer cell capable of initiating tumour growth in immunodeficient mice. Nature. 2007:445(7123):106-10.

161. Kim CF, et al. Identification of bronchioalveolar stem cells in normal lung and lung cancer. Cell. 2005;121(6):823-35.

162. Lee CJ, Dosch J, Simeone DM. Pancreatic cancer stem cells. J Clin Oncol. 2008;26(17):2806-12.

163. Li C, et al. Identification of pancreatic cancer stem cells. Cancer Res. 2007;67(3):1030-7.

164. Garg M. MicroRNAs, stem cells and cancer stem cells. World J Stem Cells. 2012;4(7):62-70.

165. Navarro A, Monzo M. MicroRNAs in human embryonic and cancer stem cells. Yonsei Med J. 2010;51(5):622-32

166. Nalls D, et al. Targeting epigenetic regulation of miR-34a for treatment of pancreatic cancer by inhibition of pancreatic cancer stem cells. PLoS One. 2011;6(8):e24099.

167. Wu MY, et al. MiR-34a regulates therapy resistance by targeting HDAC1 and HDAC7 in breast cancer. Cancer Lett. 2014;354(2):311-9.

168. Bao B, et al. Metformin inhibits cell proliferation, migration and invasion by attenuating CSC function mediated by deregulating miRNAs in pancreatic cancer cells. Cancer Prev Res (Phila). 2012;5(3):355-64.

169. Shimono $Y$, et al. Downregulation of miRNA-200c links breast cancer stem cells with normal stem cells. Cell. 2009;138(3):592-603.

170. Lee $\mathrm{Tl}$, et al. Control of developmental regulators by Polycomb in human embryonic stem cells. Cell. 2006;125(2):301-13.

171. Iliopoulos D, et al. Loss of miR-200 inhibition of Suz12 leads to polycombmediated repression required for the formation and maintenance of cancer stem cells. Mol Cell. 2010;39(5):761-72.

172. Wang $Z$, et al. The role of notch signaling pathway in epithelial-mesenchymal transition (EMT) during development and tumor aggressiveness. Curr Drug Targets. 2010;11(6):745-51.

173. Kong D, et al. Epithelial to mesenchymal transition is mechanistically linked with stem cell signatures in prostate cancer cells. PLoS One. 2010;5(8):e12445.

174. Wu Q, et al. Emerging roles of PDGF-D in EMT progression during tumorigenesis. Cancer Treat Rev. 2013:39(6):640-6.

175. Bao B, et al. Over-expression of FoxM1 leads to epithelial-mesenchymal transition and cancer stem cell phenotype in pancreatic cancer cells. J Cell Biochem. 2011;112(9):2296-306.

176. Han F, et al. Emerging roles of MicroRNAs in EGFR-targeted therapies for lung Cancer. Biomed Res Int. 2015;2015:672759.

177. Konno $Y$, et al. MicroRNA-101 targets EZH2, MCL-1 and FOS to suppress proliferation, invasion and stem cell-like phenotype of aggressive endometrial cancer cells. Oncotarget. 2014;5(15):6049-62.

178. Yang MY, et al. Nuclear localization signal-enhanced polyurethane-short branch Polyethylenimine-mediated delivery of let-7a inhibited Cancer stemlike properties by targeting the 3'-UTR of HMGA2 in anaplastic astrocytoma. Cell Transplant. 2015;24(8):1431-50.

179. Jung DE, et al. Differentially expressed microRNAs in pancreatic cancer stem cells. Pancreas. 2011;40(8):1180-7.

180. Chen J, et al. miR-125b inhibitor enhance the chemosensitivity of glioblastoma stem cells to temozolomide by targeting Bak1. Tumour Biol. 2014;35(7):6293-302

181. Hasegawa S, et al. MicroRNA-1246 expression associated with CCNG2mediated chemoresistance and stemness in pancreatic cancer. $\mathrm{Br} J$ Cancer. 2014;111(8):1572-80

182. Giovannetti E, et al. MicroRNA-21 in pancreatic cancer: correlation with clinical outcome and pharmacologic aspects underlying its role in the modulation of gemcitabine activity. Cancer Res. 2010;70(11):4528-38.

183. Park JK, et al. Antisense inhibition of microRNA-21 or -221 arrests cell cycle, induces apoptosis, and sensitizes the effects of gemcitabine in pancreatic adenocarcinoma. Pancreas. 2009:38(7):e190-9.

184. Ali S, et al. Gemcitabine sensitivity can be induced in pancreatic cancer cells through modulation of miR-200 and miR-21 expression by curcumin or its analogue CDF. Cancer Res. 2010;70(9):3606-17.

185. Li Y, et al. miR-146a suppresses invasion of pancreatic cancer cells. Cancer Res. 2010;70(4):1486-95.
186. Phuah NH, Nagoor NH. Regulation of MicroRNAs by natural agents: new strategies in Cancer therapies. Biomed Res Int. 2014;2014:804510.

187. Casey MC, et al. Exploring circulating micro-RNA in the neoadjuvant treatment of breast cancer. Int J Cancer. 2016;139(1):12-22.

188. Gu X, et al. Circulating microRNA-451 as a predictor of resistance to neoadjuvant chemotherapy in breast cancer. Cancer Biomark. 2016;16(3): 395-403.

189. Chen WX, et al. MicroRNAs delivered by extracellular vesicles: an emerging resistance mechanism for breast cancer. Tumour Biol. 2014:35(4):2883-92.

\section{Ready to submit your research? Choose BMC and benefit from:}

- fast, convenient online submission

- thorough peer review by experienced researchers in your field

- rapid publication on acceptance

- support for research data, including large and complex data types

- gold Open Access which fosters wider collaboration and increased citations

- maximum visibility for your research: over $100 \mathrm{M}$ website views per year

At $\mathrm{BMC}$, research is always in progress.

Learn more biomedcentral.com/submissions 